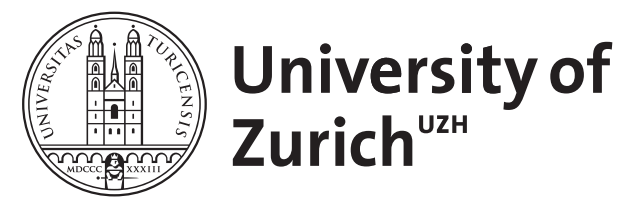

\title{
Exploring interactive furniture with EmotoCouch
}

\author{
Mennicken, Sarah ; Bernheim Brush, A J ; Roseway, Asta ; Scott, James
}

\begin{abstract}
People respond emotionally to other people, animals, or even objects like furniture. While current furniture is static in appearance, embedded electronics can enable furniture to change its appearance. A couch could show excitement during a party or anger when a pet scratches it. But would emotional furniture delight or annoy people? To explore the potential for emotional furniture, we built EmotoCouch. Through colored light, visual patterns, and haptic feedback, EmotoCouch expresses six emotional states: Excited, Happy, Calm, Depressed/Sad, Afraid, and Angry. This video describes the construction of EmotoCouch, feedback gathered through surveys and user interviews, and shows example EmotoCouch usage situations.
\end{abstract}

DOI: https://doi.org/10.1145/2638728.2638846

Posted at the Zurich Open Repository and Archive, University of Zurich

ZORA URL: https://doi.org/10.5167/uzh-102852

Conference or Workshop Item

Originally published at:

Mennicken, Sarah; Bernheim Brush, A J; Roseway, Asta; Scott, James (2014). Exploring interactive furniture with EmotoCouch. In: UbiComp Adjunct '14, Seattle, WA, USA, 13 September 2014 - 17 September 2014. s.n., 1-4.

DOI: https://doi.org/10.1145/2638728.2638846 


\section{Exploring Interactive Furniture with EmotoCouch}

\author{
Sarah Mennicken \\ University of Zurich \\ Binzmuhlestr. 14 \\ CH-8050 Zurich, Switzerland \\ mennicken@ifi.uzh.ch \\ A.J. Bernheim Brush \\ Microsoft Research \\ 1 Microsoft Way \\ Redmond, WA 98052 USA \\ ajbrush@microsoft.com \\ Asta Roseway \\ Microsoft Research \\ 1 Microsoft Way \\ Redmond, WA 98052 USA \\ ajbrush@microsoft.com \\ Permission to make digital or hard copies of part or all of this work for \\ personal or classroom use is granted without fee provided that copies are \\ not made or distributed for profit or commercial advantage and that \\ copies bear this notice and the full citation on the first page. Copyrights \\ copies bear this notice and the full citation on the first page. Copyrights
for third-party components of this work must be honored. For all other \\ for third-party components of this
uses, contact the Owner/Author \\ Copyright is held by the owner/author(s). \\ UbiComp '14 Adjunct, September 13-17 2014, Seattle, WA, USA \\ ACM 978-1-4503-3047-3/14/09. \\ http://dx.doi.org/10.1145/2638728.2638846 \\ James Scott \\ Research \\ jws@microsoft.com \\ 21 Station Rd
}

\begin{abstract}
People respond emotionally to other people, animals, or even objects like furniture. While current furniture is static in appearance, embedded electronics can enable furniture to change its appearance. A couch could show excitement during a party or anger when a pet scratches it. But would emotional furniture delight or annoy people? To explore the potential for emotional furniture, we built EmotoCouch. Through colored light, visual patterns, and haptic feedback, EmotoCouch expresses six emotional states: Excited, Happy, Calm, Depressed/Sad, Afraid, and Angry. This video describes the construction of EmotoCouch, feedback gathered through surveys and user interviews, and shows example EmotoCouch usage situations.
\end{abstract}

\section{Author Keywords}

Emotions; furniture; couch; domestic environment; haptic feedback; embedded hardware; color.

\section{ACM Classification Keywords}

H.5.m. Information interfaces and presentation (e.g., HCI): Miscellaneous.

\section{Introduction}

Expressing and responding to emotions is an important part of people's lives. People respond emotionally to other people, animals, or even objects. Current 
furniture can elicit an emotional response through its shape, use of textile, or color; however, these characteristics are static and unchanging. But what if furniture could change its appearance based on the surroundings? Perhaps even express emotions itself?

A couch that can change its appearance and has its own emotions has the potential to play many different roles in a home; delighting kids with a new appearance when they come home, offering comfort by changing to a preferred design when someone is sad, showing an angry design to highlight tension when people are arguing, or showing an excited design to subtly encourage family time together when a family shares the couch.

To study people's reactions to an emoting couch, we selected emotions from each quadrant in the Circumplex model of emotions [4]. The six emotions we chose for the couch to convey were: afraid, angry, calm, depressed/sad, excited, and happy (see Figure 1).
Using research conducted in the field of psychology to explore people's associations of colors $[1,6]$ and patterns [5] with emotions, we selected a color and pattern for each emotion. The combined looks designed for each emotion are shown in Figure 2.
Figure 1. Chosen set of emotions in the Circumplex model

\footnotetext{
Figure 2. Color/pattern combinations for six
} implemented emotions. 
Based on research associating physical movements to emotions [3] we also designed different haptic behaviors for each emotion, e.g., a steady, repetitive, mediumpaced drum beat for "happy" and a very irregular, fast one for "afraid".

\section{Building EmotoCouch}

To build EmotoCouch we purchased an IKEA KARLSTAD couch frame and built four custom cushions. The cushions each contain 160 embedded, individually controllable RGB LEDs for a total of 640 lights overall and an embedded voice coil (see Figure 3 ). Using the LEDs the couch's color can be changed and by playing subsonic sounds using the voice coil the person sitting on the couch experiences haptic feedback.

We created different patterns on the cushion covers for each emotion. For prototyping, we hand-drew 6 different patterns on custom covers, one for each emotion (see Figure 2). However, in parallel we also experimented with methods to dynamically change the pattern showing on the couch. As illustrated in Figure 4 , we stitched thin nichrome wire through fabric in distinct patterns using a mix of screencast and thermochromic pigments. When current is applied to heat the nichrome wires, the pigments become clear making the pattern disappear.

Figure 5 shows the EmotoCouch in a living room setup. We conducted a lab study with EmotoCouch to gather feedback from parents and children on the concept of emoting furniture and whether the couch's emotions are consistently identified by different people interacting with the couch. Participants shared their reactions to furniture with its own emotions and told us when and for what reason they would want their furniture to change its appearance, such as excitement during a party and happiness when family members are together. Several of these situations are illustrated in the video.

\section{Concluding Remarks}

We built EmotoCouch, a furniture prototype that displays emotions using lights, patterns, and haptics, to explore the concept of interactive furniture. Participants who interacted with EmotoCouch were intrigued by the concept and suggested many possible usage situations. Opinions differed on whether the couch should have its 
Figure 5. EmotoCouch in a living room setup.

own emotions and proactively switch between them or should be set manually to a desired emotion by residents. The video shows how we built EmotoCouch, gathered feedback and illustrates potential usage situations.

Moving forward we are expanding the range of embedded sensors and actuators as well as connecting EmotoCouch to the Lab of Things SDK [2]. This integration simplifies deployment into people's homes for in-situ studies and enables changing the emotion displayed by EmotoCouch based on data collected by other in-home sensors.

\section{Acknowledgements}

We thank Scott Saponas and Jan Kučera for their technical assistance.

\section{References}

[1] Hupka, R. B., Zaleski, Z., Otto, J., Reidl, L. and Tarabrina, N. V., The colours of anger, envy, fear, and jealousy. a cross-cultural study, Journal of crosscultural psychology 28, 2 (1997), 156-71.

[2] Lab of Things, http://www.lab-of-things.com/

[3] Lee, J.-H., Park, J.-Y., and Nam, T.-J., Emotional Interaction Through Physical Movement. In Proc. HCII 2007.

[4] Russell, J.A., A circumplex model of affect. Journal of Personality and Social Psychology 39, 6(1980), 1161-1178.

[5] Ståhl, A., Sundström, P. and Höök, K., A foundation for emotional expressivity. In Proc. DUX 2005.

[6] Terwogt, M.M. and Hoeksma, J. B., Colors and Emotions: Preferences and Combinations, Journal of General Psychology 122, 1 (1995), 5-17. 